\title{
Caracterización Clínica y Epidemiológica de Dengue Hemorrágico en Neiva, Colombia, 2004
}

\author{
Clinical and epidemiological characterisation of dengue \\ haemorrhagic fever in Neiva, Colombia, 2004
}

Doris M. Salgado ${ }^{1}$, Jairo A. Rodríguez², Marisol Garzón ${ }^{1}$, Gerson Cifuentes², Milton Ibarra 2 , Martha R. Vega ${ }^{1}$ y Dolly Castro ${ }^{2}$

1. Universidad Surcolombiana. Hospital Universitario de Neiva, Colombia. domasal59@yahoo.com, magalo@ixp.net, martharociovega@hotmail.com

2. Universidad Surcolombiana, Neiva, Colombia. jrodriguez@usco.edu.co, miltonibarra@latinmail.com, dollycastro@usco.edu.co

Recibido 12 Junio 2006/Enviado para Modificación 19 Noviembre 2006/Aceptado 3 Diciembre 2006

\section{RESUMEN}

Objetivo Neiva es considerada endémica para fiebre dengue y fiebre dengue hemorrágico en Colombia. Durante el 2004 se presentó un brote epidémico, siendo necesario caracterizar el comportamiento epidemiológico y clínico durante esta epidemia.

Materiales y Métodos Estudio retrospectivo descriptivo de niños menores de 13 años que ingresaron al Hospital Universitario en este periodo, con criterios de Fiebre dengue y Fiebre dengue hemorrágico según la OMS. Se registraron los datos demográficos, clínicos y paraclínicos de ingreso. El análisis fue descriptivo y bivariado exploratorio utilizando la prueba de $\mathrm{chi}^{2}$.

Resultados De 105 niños el 87,6 \% ingresó con diagnóstico de fiebre dengue hemorrágico y el 12,4\% con fiebre dengue. Un $67 \%$ correspondió a menores de 5 años, con mayor frecuencia y severidad en niñas. El $83 \%$ ingresó en los primeros seis días de enfermedad. Hubo datos de choque en el $20 \%$ de los pacientes, de estos el $76 \%$ presentó complicaciones (chi ${ }^{2} 29.53, \mathrm{gl} \mathrm{6,} \mathrm{p=0.0000).} \mathrm{Entre} \mathrm{los} \mathrm{datos}$ paraclínicos, las aminotransferasas se encontraron 3 a 5 veces el valor normal y hubo correlación entre recuento plaquetario inferior a 20000 por $\mathrm{mm}^{3}$ y choque al ingreso (chi ${ }^{2}$ 20,65, gl 4, p= 0.0004). La evolución fue favorable; sin embargo, hubo complicaciones en el $32 \%$ de los casos (13\% miocarditis, $19 \%$ hepatitis o encefalitis y $2 \%$ sepsis).

Conclusión Las características epidemiológicas y clínicas observadas en esta cohorte de pacientes mostraron una variación en género, edad y órgano blanco, con una incidencia alta de compromiso miocárdico.

Palabras Clave: Fiebre dengue, dengue hemorrágica, dengue, miocarditis, hepatitis viral humana (fuente: DeCS, BIREME). 


\begin{abstract}
Objective Neiva (a southern Colombia city) is endemic for dengue fever and dengue haemorrhagic fever. Neiva has suffered outbreaks of dengue, that in 2004 being the latest one. This study was designed to characterise epidemiological and clinical data from that outbreak of dengue.

Material and Methods This was a descriptive, retrospective study of children aged less than 13 years who were admitted to the University Hospital and fulfilled the WHO's clinical and laboratory criteria for dengue or dengue haemorrhagic fever. Demographic, clinical and paraclinical data were recorded and analysed using Chi square $\left(\mathrm{Chi}^{2}\right)$ bivariate tabular test.

Results 105 children were diagnosed as suffering from either dengue haemorrhagic fever $(87.6 \%)$ or dengue fever (12.4\%); $67 \%$ of them were aged less than 5 years. Girls were more frequently affected by severe clinical manifestations. $83 \%$ of the children were admitted during the first six days of the disease; dengue shock syndrome was diagnosed in $20 \%$ and $76 \%$ presented clinical complications (Chi ${ }^{2} 29.53$, gl 6, p=0.0000). Aminotransferases were 3 to 5 times above normal levels. There was a statistical correlation between low platelet count (less than 20000 per $\mathrm{mm}^{3}$ ) and shock during admission (Chi $20.65, \mathrm{gl} \mathrm{4,} \mathrm{p}=0.0004)$. Complications arose during clinical evolution in $32 \%$ of the cases (13\% myocarditis, $19 \%$ hepatitis or encephalitis and $2 \%$ sepsis). Conclusion The clinical and epidemiological characteristics observed in this cohort evidenced differences in age, gender and organs affected compared to data described in the literature; there was a high incidence of myocarditis.
\end{abstract}

Key Words: Dengue fever, dengue hemorrhagic fever, myocarditis, human viral hepatitis (source: $\mathrm{MeSH}, \mathrm{NLM}$ ).

$\mathrm{E}$ l dengue es la principal enfermedad viral transmitida por artrópodos en el mundo (1). El virus dengue es miembro de la familia Flaviviridae, virus RNA, envuelto con 4 serotipos DEN1-4, todos ellos transmitidos al ser humano por un mosquito del género Aedes, principalmente el Aedes aegypti para las Américas (2); es altamente domiciliariario y tiene preferencia por picar a los humanos (3).

La enfermedad se caracteriza por un cuadro febril agudo que compromete el estado general, asociado a cefalea, mialgias y leucopenia , la fiebre dengue (FD) es autolimitada en general, sin embargo, existe el riesgo de presentar en el curso de la evolución la forma complicada conocida como fiebre dengue hemorrágico (FDH) cuyas manifestaciones se relacionan con alteraciones de la hemostasia e incremento de la permeabilidad vascular que puede llevar al paciente al choque hipovolémico ( SSD) y a la muerte si no se instaura un adecuado manejo (3-4). 
Su incidencia ha crecido en las áreas tropicales y subtropicales en forma rápida pasando de pocos países en los años 50 a más de 100 países en el 2000. La Organización Mundial de la Salud (OMS) reporta 50 millones de casos de FD cada año y cerca de 500000 casos de FDH con una mortalidad que oscila entre el 1 y 4,5 \% (1) El incremento dramático de la enfermedad especialmente en los últimos 30 años la ha convertido en un verdadero problema de salud pública cuya expansión a la fecha no ha podido ser controlada.Esta misma tendencia se ha observado en países de Latinoamérica como Venezuela, Brasil, Cuba y Colombia. En Colombia la incidencia pasó de menos de 100 casos en 1990 a 57956 casos de fiebre dengue y de 0 a 5171 casos de FDH notificados en 1998 (5-6).

El Huila aparece junto a Santander y Valle del Cauca como una de las regiones de mayor endemicidad para FD y FDH. Los primeros casos de FDH se reportaron en 1991, a partir de esa fecha los reportes han ido aumentando progresivamente año tras año y se han documentado dos epidemias, la ultima en 1997 (7-9). Sin embargo, el año 2004 mostró un claro comportamiento epidémico con una tasa de 60 por 100000 mientras el promedio nacional fue de 12 por 100 000. Esto tuvo un gran impacto social y económico con grandes tasas de hospitalización e incremento en costos hospitalarios (10-11).

En Neiva está el Hospital Universitario Hernando Moncaleano Perdomo, hospital de $3^{\circ}$ y $4^{\circ}$ nivel de atención y centro de referencia de la región Surcolombiana, que involucra los departamentos de Huila, sur del Tolima, Caquetá y Putumayo. Además, es el lugar de atención del mayor número de pacientes con FDH en la región. El objetivo de este estudio es describir las características epidemiológicas y clínicas de los pacientes durante esta epidemia con el fin de hacer un análisis y establecer factores pronóstico.

\section{MATERIALES Y MÉTODOS}

El diseño del estudio fue descriptivo, retrospectivo, basado en los datos de las historias clínicas de los pacientes menores de 13 años, hospitalizados en el servicio de pediatría del Hospital Universitario de Neiva durante el período de 1 Enero al 30 de Junio de 2004. La población de estudio fueron todos los pacientes niños con los criterios de la OMS: fiebre alta entre dos y siete días, manifestaciones hemorrágicas (mínimo prueba de torniquete positiva), recuento plaquetario menor de 100000 por $\mathrm{mm}^{3}$, manifestaciones de fuga vascular (hemoconcentración mayor a 20 \%, ascitis, o efusión pleural), IgM sérica 
positiva para virus dengue, choque (tensión arterial sistólica menor al percentil 5 para la edad y presión de pulso menor a $30 \mathrm{mmHg}$ ) (1).

Para la recolección de la información se usó un formato que incluía las variables sociodemográficas, características clínicas, exámenes paraclínicos y evolución de cada paciente.

Para el procesamiento de la información se utilizó el software epi-info versión 3.02 de 2004. El análisis estadístico descriptivo, presenta los porcentajes de cada variable. Se realizó también un análisis bivariado exploratorio con algunas variables de interés, utilizando la prueba de Chicuadrado $\left(\mathrm{Chi}^{2}\right)$. El estudio fue presentado y aprobado por el comité de ética del Hospital.

\section{RESULTADOS}

Durante el primer semestre de 2004 se registró un total de 105 pacientes atendidos en el servicio de pediatría de los cuales 87,6 \% ingresaron con diagnóstico de FDH y un 12,4 \% con FD. Las características sociodemográficas y clínicas de ingreso se muestran en la Tabla 1. En la distribución por edad, el $67 \%$ de los pacientes fueron menores de 5 años, con un número importante de pacientes lactantes menores de 1 año (20\%). No hubo mayores diferencias respecto al género sin embargo, un análisis bivariado exploratorio mostró una mayor frecuencia de enfermedad en niñas en el grupo menor de 10 años y más frecuente en niños en el grupo mayor a 10 años $\left(\mathrm{Chi}^{2}{ }^{10.63}\right.$, gl 4, p=0.03).

En cuanto a la procedencia geográfica, tres de cada cuatro pacientes hospitalizados procedían del área urbana de Neiva, 30,4\% de los casos de la comuna 9 (Norte, Galindo, Luís Ignacio Andrade, Luís Carlos Galán)

Cerca de un $83 \%$ ingresaron en los primeros seis días de enfermedad, en período de defervescencia. El estado nutricional de los pacientes evaluado con base en la relación peso para la edad, mostró porcentajes similares de pacientes eutróficos y de bajo peso.

Se encontró datos de choque en un $20 \%$ de los pacientes que coincide con el porcentaje de pacientes con llenado capilar lento y presión de pulso estrecha. De los pacientes que presentaron choque con presión de pulso estrecha (menor de 30 mmHg), el 76 \% tuvo algún tipo de complicación (24 \% con miocarditis, el $48 \%$ con otra complicación y un $4 \%$ con sepsis), mientras que los pacientes 
con presión de pulso normal al ingreso sólo el 19,2 \% presentó algún tipo de complicación. ( $\mathrm{Chi}^{2}$ 29,53, gl 6, p=0.0000).

Tabla 1. Características demográficas y clínicas al ingreso de los niños con diagnóstico Fiebre Dengue - Fiebre Dengue Hemorrágica del Hospital

Universitario de Neiva. 2004

\begin{tabular}{|c|c|c|}
\hline $\begin{array}{c}\text { Características } \\
\text { sociodemográficas y clínicas }\end{array}$ & $\begin{array}{r}\text { Frecuencia } \\
n=105\end{array}$ & Porcentaje \\
\hline \multicolumn{3}{|l|}{ Género } \\
\hline Femenino & 59 & 56,2 \\
\hline Masculino & 46 & 43,8 \\
\hline \multicolumn{3}{|l|}{ Edad } \\
\hline Menor de 6 meses & 5 & 4,8 \\
\hline 6 a 11 meses & 8 & 7,6 \\
\hline 1 a 4 .años 11 meses & 57 & 54,3 \\
\hline 5 a 10 años & 22 & 21,0 \\
\hline mayor de 10 años & 13 & 12,4 \\
\hline \multicolumn{3}{|l|}{ Tensión arterial sistólica (TAS) } \\
\hline$<$ Percentil 5 & 13 & 12,4 \\
\hline Normal & 92 & 87,6 \\
\hline \multicolumn{3}{|l|}{ Presión de pulso (mmHg) } \\
\hline ? $\quad 30$ & 26 & 24,8 \\
\hline 30 & 79 & 75,2 \\
\hline \multicolumn{3}{|l|}{ Hepatomegalia } \\
\hline Sin hepatomegalia & 8 & 7,6 \\
\hline Con hepatomegalia & 86 & 81,9 \\
\hline Sin datos & 11 & 10,5 \\
\hline \multicolumn{3}{|l|}{ Sangrado } \\
\hline Epistaxis & 16 & 15,2 \\
\hline Gingivorragia & 2 & 1,9 \\
\hline Hematemesis y melenas & 11 & 10,5 \\
\hline Petequias & 37 & 35,2 \\
\hline No hemorragia & 26 & 24,8 \\
\hline Sin dato & 13 & 12,4 \\
\hline
\end{tabular}

Con relación a los hallazgos paraclínicos que se muestran en la Tabla 2, el $95 \%$ de los pacientes tuvieron recuentos plaquetarios por debajo de 100000 por $\mathrm{mm}^{3} \mathrm{sin}$ embargo, todos los pacientes con recuento de plaquetas inferior a 20000 por $\mathrm{mm}^{3}$ ingresaron con diagnóstico de Síndrome de choque dengue (SSD) (Chi² 20,65, gl 4, p= 0.0004). Se observó hemoconcentración en el 25 $\%$ de los pacientes. El nivel de aminotransferasas fue evaluado en el $68 \%$ de los casos encontrándose elevado entre 2 y 5 veces su valor normal en dos terceras partes de las muestras y una tercera parte tuvo incrementos de más de 5 veces.

Otras variables importantes en el análisis son las relacionadas con el tratamiento. El $21 \%$ de los pacientes requirió la administración de cristaloides, el $18 \%$ coloides (90 \% recibió un bolo) y el 41 \% soporte inotrópico. De los 
pacientes a quienes se les administró albúmina un 80 \% tenían derrame pleural mayor del $30 \%$. Con el suministro de albúmina no se encontró diferencia estadísticamente significativa entre la presión del pulso, cifras de tensión arterial sistólica y tiempo de recuperación.

Tabla 2. Características bioquímicas y hematológicas al ingreso de los pacientes con Fiebre Dengue - Fiebre Dengue Hemorrágica del Hospital Universitario de Neiva. Primer semestre de 2004.

\begin{tabular}{lrr}
\hline \multicolumn{1}{c}{ Características } & $\begin{array}{r}\text { Frecuencia } \\
\mathrm{n}=105\end{array}$ & Porcentaje \\
\hline Hemoconcentración (\%) & 71 & 67,6 \\
$<\quad 20 \%$ & 25 & 23,8 \\
$>\quad 21 \%$ & 19 & 18,1 \\
sin dato & & \\
Plaquetas por $\mathrm{m} \mathrm{m}^{3}$ & 10 & 9,5 \\
$<\quad 20.000$ & 63 & 60,0 \\
$20.000-50.000$ & 29 & 27,6 \\
$50.000-100.000$ & 1 & 1,0 \\
Sin dato $\quad 2000$ & 2 & 1,9 \\
$\quad$ & \\
Aminotransferasas (TGO & & \\
- GPT) UI/ dl & & \\
Normal & & \\
40 a 100 & 5 & 4,8 \\
100 a 200 & 15 & 14,3 \\
mayor de 200 & 34 & 32,4 \\
Sin dato & 17 & 16,2 \\
& 34 & 32,4 \\
\hline
\end{tabular}

La evolución de los pacientes se muestra en la Tabla 3. La mayoría de los pacientes evolucionaron favorablemente, con una recuperación entre el $3^{\text {er }} \mathrm{y}$ $5^{\circ}$ día de hospitalización. En el 32 \% se evidenció alguna complicación: 13 \% de miocarditis, un $19 \%$ otro tipo de complicación como hepatitis o encefalitis y un $2 \%$ sepsis. Al analizar las características demográficas y la presencia de complicaciones, se encontró que estas fueron más frecuentemente en las niñas (OR 3,36 IC 95 \% 1,11- 10,63; Chi 5,77, gl 1, p 0,016).

Teniendo en cuenta las características de las variables, se realizó un análisis exploratorio utilizando un modelo de regresión logística, con aquellas variables que dieron resultado significativo en el análisis bivariado. Se plantearon inicialmente los modelos con las variables género, presión de pulso, plaquetas, como variable resultado la presencia de complicaciones. La única variable que mostró una posible asociación estadística con las complicaciones fue la presión de pulso OR 13,98 IC $95 \%(4,6$ - 42,49) $\mathrm{p}=0.0000$. Se puede observar que los pacientes con presión de pulso menor de $30 \mathrm{mmHg}$ tuvieron 14 veces más riesgo de complicaciones, siendo el resultado estadísticamente significativo. 
Tabla 3. Evolución de los pacientes con Fiebre Dengue - Fiebre Dengue Hemorrágica del Hospital Universitario de Neiva. Primer semestre de 2004

\begin{tabular}{lrr}
\hline \multicolumn{1}{c}{$\begin{array}{c}\text { Características de } \\
\text { evolución }\end{array}$} & $\begin{array}{r}\text { Frecuencia } \\
\mathrm{N}=105\end{array}$ & Porcentaje \\
\hline Miocarditis & 13 & 12,4 \\
Sobre infección & 2 & 1,9 \\
Otra & 19 & 18,1 \\
No complicaciones & 69 & 65,7 \\
Sin dato & 2 & 1,9 \\
Persistencia de Síndrome & & \\
de Choque Dengue & 24 & 22,9 \\
Si & 81 & 77,1 \\
No & & \\
Presencia de Re- choque & & \\
Si & 15 & 14,3 \\
No & 88 & 83,8 \\
Sin dato & 2 & 1,9 \\
Recuperación & & \\
12 a 24 horas & 5 & 4,8 \\
$25-48$ horas & 10 & 9,5 \\
$49-72$ horas & 34 & 32,4 \\
73 - 96 horas & 24 & 22,9 \\
$>96$ horas & 32 & 30,5 \\
\hline
\end{tabular}

\section{DISCUSIÓN}

Revisiones sobre incidencia de FD y FDH en diferentes grupos etareos han mostrado un desplazamiento hacia poblaciones entre los 15 y 39 años (12). Tal fenómeno ha sido observado en el sureste asiático y en Centro América, en regiones de alta endemicidad como Cuba y Puerto Rico. Sin embargo, este comportamiento no se observó en la población de este estudio en el cual el grupo de niños menores de 5 años sigue siendo el más afectado (54,3 \%). Este resultado contrasta con el encontrado en otro estudio de población colombiana en donde al analizar 195 niños la FDH predominó en la población escolar (6 a 12 años) con el 49,3 \% (13).

Respecto a la distribución de la enfermedad por género, siempre se ha visto una mayor incidencia en hombres, con relaciones que oscilan entre 2,5 -1,5: 1 hombre: mujer (3-12). En esta serie se invierte esa relación a 0,8: 1 hombre: mujer, con un mayor número de complicaciones en las niñas ( $p$ 0.016), lo cual coincide con lo descrito en otras regiones como en Bucaramanga, Colombia donde la prevalencia fue predominante en el sexo masculino (51,2 \%) (14), Bangladesh y Centro América (12). Desde los años 70, Halstead ya 
había planteado que esta observación se debía a una mejor capacidad de respuesta inmune ante el virus y por ende mayores posibilidades de complicaciones.

Aunque la procedencia de los pacientes fue en su mayoría del área urbana de Neiva, es de resaltar que existen asentamientos poblacionales rodeando el perímetro urbano con características rurales, cuyos pacientes se atienden en el área urbana. La ruralización de la enfermedad se vería facilitada por estas condiciones de suburbanismos vistas como consecuencia del desplazamiento y pobreza. Esto ha sido también tenido en cuenta en estudios asiáticos.

El concepto tradicional de la relación entre el buen estado nutricional y la presentación de FDH ha sido referido en estudios en Bangladesh y en Puerto Rico (12), contrario a lo observado en las epidemias en India, donde no hubo relación entre estado nutricional y la severidad del dengue. El mismo comportamiento se observó en esta serie, en la cual el 50 \% de los pacientes tenían bajo peso para la edad, reflejo del estado nutricional precario de nuestra población. Es importante destacar este cambio en el perfil del niño que puede ser el blanco de la enfermedad; los mejor nutridos o eutróficos que tradicionalmente habían sido referidos como un factor dependiente del huésped para las complicaciones, no se cumple en esta serie y por el contrario pone de presente que cualquier niño independiente de su estado nutricional puede hacer formas severas y mortales de enfermedad tipo dengue.

De las variables hemodinámicas de interés, se resalta el valor de la presión de pulso como herramienta útil en el diagnóstico de choque y su correlación estadísticamente significativa con la presencia de complicaciones ( $\mathrm{p}=0.0000)$, lo que valida esta herramienta como factor pronóstico.

La hepatomegalia fue un signo constante en los pacientes, con elevación de las aminotransferasas en una relación 1,43 AST /ALT lo que sugiere alteración en la función hepática. Este hallazgo ha sido descrito en otros estudios realizados en el sudeste asiático, a partir de los cuales se identifica al hepatocito como célula blanco para virus dengue, jugando un papel definitivo en las formas clínicas severas y llevando a los pacientes a diferentes grados de hepatitis hasta su forma extrema con falla hepática fulminante (15-19).

La disfunción hepática facilita otro fenómeno que es la alteración en la hemostasia, lo que agrava las hemorragias de los pacientes con dengue $(18,20)$. Así mismo, en un estudio realizado en Santander, una región al nororiente de 
Colombia, predominó la complicación hepática (27 \%), seguido de la neurológica (25 \%) (13). Lo que resalta la capacidad del virus de afectar la célula hepática en ciertos pacientes.

En este estudio, las hemorragias fueron vistas en el 75\% de los casos la mayoría en piel y mucosas diferentes al tracto gastrointestinal; esto contrasta con un estudio nacional en el Valle, en el cual el sangrado más frecuente se presentó en el tracto digestivo (21). Diferentes estudios muestran correlación entre severidad y recuento plaquetario bajo $(16,18)$ similar a lo visto en los pacientes del estudio, en los cuales hubo una correlación estadísticamente significativa en el análisis bivariado entre choque y plaquetas menor de 20 $000 / \mathrm{mm} 3\left(\mathrm{Chi}^{2}\right.$ 20,65, gl 4, p= 0.0004). Más aún, en un reciente estudio en población adulta colombiana se observó una importante correlación entre trombocitopenia profunda (menor de $50000 / \mathrm{mm}^{3}$ ) con la severidad de la enfermedad, convirtiendo tal signo en un potencial factor pronóstico (22).

En el manejo de los pacientes se han planteado controversias del beneficio del uso de coloides sobre los cristaloides $(23,24)$. Nuevamente, al igual que en otras series, no se pudo establecer ventajas con el uso de coloides respecto a la rapidez de resolución del choque como tampoco sobre la estancia hospitalaria.

El compromiso de órgano blanco en infección por virus dengue ha mostrado variación geográfica y virológica, es así como en Tailandia y Vietnam hay un alto reporte de hepatitis dengue mientras en Filipinas han descrito casos de encefalitis dengue $(25,26)$. De manera análoga, en la región Surcolombiana, en diferentes cohortes, se ha encontrado compromiso miocárdico de diferente severidad incluida la miocarditis fulminante. En esta serie en particular, nuevamente aparece la miocarditis en 13 casos (13\%) considerada una incidencia muy alta si se compara con la de las demás series. Al momento, el estudio de la miocarditis asociada a virus dengue, se ha limitado al reporte de casos en regiones como India principalmente $(27,28)$.

Esta serie muestra el carácter epidémico de la enfermedad en nuestra región, con una desviación en género y ruralización, así como también la aparición del hígado como importante órgano blanco en dengue

Dedicatoria. Dedicamos este trabajo a la familia de Nuestro colega Gerson Danilo Cifuentes, cuya dedicación y participación entusiasta, durante el desarrollo de este proyecto fueron el reflejo de su decidido compromiso con la Pediatría y el amor por 
los niños demostrado a lo largo de su vida, que llegó a su fin unos meses después de concluido este trabajo.

\section{REFERENCIAS}

1. World Health Organization (WHO). Dengue and Dengue Haemorrhagic Fever. Fact. Sheet. Geneva 2002; 117- 119.

2. Gubler DJ. Dengue and dengue hemorrhagic fever. Clin Microbiol Rev 1998. 11; 3:480- 96.

3. Baush DG. Viral Hemorrhagic fevers in the Americas. Clin Lab Med 2002; 22: 981-1020.

4. Kautner I. Dengue virus infection: Epidemiology, Pathogenesis, Clinical presentation, Diagnosis and Prevention. J Pediatr 1997; 13: 516-5

5. Instituto Nacional de Salud. Subdirección de Epidemiología y Laboratorio Nacional de Referencia. Ministerio de Salud. Bogotá: Boletín epidemiológico 2002; 7: 189-91.

6. Rodríguez CP. Estrategias para el control del Dengue y el Aedes aegypti en las Américas. Rev Cubana Med Trop 2002; 54:189-201.

7. Secretaría de Salud Departamental. Análisis de la morbilidad y mortalidad- dengue. Boletín Epidemiológico Huila 2003; 2: 89-93.

8. Salgado D, Rodríguez J, Vega R. Dengue hemorrágico emergencia pediátrica en el Huila. Pediatría 1997; 34: 78-83.

9. Fajardo P. Nociones populares sobre "Dengue" y "Rompe huesos" dos modelos de enfermedad en Colombia. Rev Panam Salud Publica 2001;10: 161-168.

10. Salgado D, Narváez C. Dengue hemorrágico. Temas pediátricos 2002; 19:5-24.

11. Valdés L. Impacto económico de la epidemia de dengue 2 en Santiago de Cuba en1997. Rev Cubana Med Trop 2002; 54: 220-27.

12. Debarati G, Schimmer B. Dengue fever: new paradigms for a changing Epidemiology. Emerg Themes Epidemiol 2005, 2: 1- 34.

13. Méndez A, González G. Manifestaciones clínicas inusuales del dengue hemorrágico en niños. Biomédica 2006; 26: 61 - 70.

14. Díaz FA, Martínez RA, Villar LA. Criterios clínicos para diagnosticar el dengue en los primeros dias de enfermedad. Biomédica 2006; 26: 22 - 30

15. Nimmannitya S. Clinical spectrum and management of dengue hemorrhagic fever. Southeast Asian J Trop Med Public Health 1987; 18:392-97.

16. Nguyen TL, Nguyen TH, Tieu NT et al. The impact of dengue haemorrhagic fever on liver function. Res Virol 1997; 148: 273-77.

17. Del Valle S. Hepatitis reactiva por virus del Dengue Hemorrágico. Rev Cubana Med Trop 2001; 1: 28-31. 
18. Lei H, Yeh T, Liu H, Lin Y, Chen S, Liu Ch. Immunopathogenesis of dengue virus Infection. J Biomed Sci 2001; 8:377-88.

19. Malavige GN, Fernando S, Fernando DJ. Dengue viral infection. Post J 2004; 80 : 588-601.

20. Krishnamurti C, Kalayanarooj S. Mechanisms of haemorrhage in Dengue without circulatory collapse. Am J Trop Med Hyg 2001; 65: 840-47

21. Rosso F. Dengue Hemorrágico en el Hospital Universitario del Valle. Colomb Med. 1994; 25: 10-20.

22. Diaz-Quijano FA, Villar-Centeno LA, Martínez-Vega RA. Complicaciones asociadas a la trombocitopenia profunda en pacientes con dengue. Rev Med Chile 2006; 134:167-173.

23. Halstead SB. Dengue. Curr Opin Infect Dis 2002; 15: 471-76.

24. Wills BA, Dung NM, Loan HT. Comparison of three fluid solutions for resuscitation in dengue shock syndrome. New Eng J Med 2005; 353: 877-88.

25. Cardosa MJ. Dengue haemorrhagic fever: questions of pathogenesis Curr Opin. Infect Dis 2000; 13: 471-75.

26. Huerre MR, Lan N, Marianeaux P, Hue NB, Khun H, Hung NT, et al. Liver histopathology and biological correlates in five cases of fatal dengue fever in Vietnamese's Children. Virchows Arch 2001; 438: 107-15.

27. Kabra SK, Juneja R. Myocardial Dysfunction in children with Dengue Haemorrhagic Fever. Natl Med J India 1998; 11: 59-61.

28. Wali JP, Biswas A. Cardiac involvement in dengue haemorrhagic fever. J Cardiol 1998; 64:31-6. 